\title{
Nicotine replacement lozenges: abuse-related hyperkeratosis of the lateral border of the tongue. A case report
}

\author{
K. B. Naudi ${ }^{1}$ and D. H. Felix ${ }^{2}$
}

A case of a white patch on the right lateral border of the tongue, found to be hyperkeratosis induced by long-term habitual placement of nicotine replacement lozenges (NiQuitin $\mathrm{CQ}^{\circledR}$ ) in the right lingual sulcus, is presented. The lesion became less evident once the patient varied the intraoral location and reduced his consumption of these tablets.

\section{INTRODUCTION}

Nicotine replacement therapy (NRT) is the most widely used pharmacological therapy for smoking cessation. ${ }^{1}$ NRT is available in a number of different formulations including chewing gum, transdermal patches, nasal sprays, mouth inhalers, lozenges, and sublingual tablets (Table 1). All forms of NRT are effective as part of a strategy to promote smoking cessation. They increase the odds of quitting approximately 1.5 to 2 fold regardless of setting. ${ }^{2}$

We present a case of hyperkeratosis on the right lateral border of the tongue caused by the habitual placement of NRT lozenges in the area, which regressed once the habit was reduced. To our knowledge this is the first case report of such an occurrence.

\footnotetext{
"Specialist Registrar in Oral Surgery, Glasgow Dental Hospital \& School, Oral Medicine, 378 Sauchiehall Street, Glasgow, G2 3JZ; ${ }^{2}$ Consultant in Oral Medicine, NHS Education for Scotland, 2nd Floor, Hanover Buildings, 66 Roe Street, Edinburgh, EH2 2NN Correspondence to: Mr Kurt Busuttil Naudi Email:kurt.naudi@northglasgow.scot.nhs.uk
}

\section{CASE REPORT}

A 53-year-old male was seen for a sixmonthly review appointment in the Oral Medicine Department, Glasgow Dental Hospital and School, in June 2005. He had a history of biopsy-proven moderate to severe epithelial dysplasia on the left floor of mouth that had been completely excised surgically in January 2002. His past medical history was otherwise unremarkable. Since the surgery he had been smoking two cigarettes per day and was using 12 lozenges of NiQuitin $\mathrm{CQ}^{\circledR}$ $2 \mathrm{mg}$ per day.

Clinical examination revealed an asymptomatic diffuse white patch on the right lateral border of the tongue (Fig. 1). The remainder of the clinical examination was otherwise unremarkable and in particular there were no other oral mucous membrane lesions.

On further questioning the patient admitted to a habit of placing the NRT lozenges in the right lingual sulcus. It was hypothesised that this could be hyperkeratosis in response to chronic chemical irritation.

\section{TREATMENT}

In view of the previous history of dysplasia, a biopsy of the white patch was performed under local anaesthesia and this was submitted for histopathological examination. This revealed heavily keratinised squamous epithelium that appeared irregularly thickened. No epithelial dysplasia was identified. The lamina propria contained focal mild non-specific chronic inflammatory infiltrate (Fig. 2). The provisional diagnosis of traumatic hyperkeratosis was confirmed.

The patient was advised of the results and encouraged to attempt reduction in the number of lozenges consumed and also to vary the location in which the lozenges were kept intraorally.

At subsequent review the patient stated that he had reduced the lozenges to ten per day and had attempted to keep the lozenges in different intraoral sites. Clinical examination showed significant reduction in the extent of the white patch on the right lateral border of the tongue (Fig. 3) and no new hyperkeratotic areas could be seen intraorally.

\section{DISCUSSION}

Nicotine replacement therapy is a widely used and well recognised aid for smoking cessation. Reported side effects of NRT lozenges include: diarrhoea, flatulence, heartburn, hiccups, nausea, upper 


\begin{tabular}{|c|c|c|}
\hline Formulation & Available concentrations & Maximum daily dose \\
\hline Lozenge & $2 \mathrm{mg}$ and $4 \mathrm{mg}$ & 15 lozenges \\
\hline Chewing gum & $2 \mathrm{mg}$ and $4 \mathrm{mg}$ & 12 tablets \\
\hline Skin Patch & $7 \mathrm{mg}, 14 \mathrm{mg}$ and $21 \mathrm{mg}$ & 1 patch \\
\hline Inhaler & 10 mg per cartridge* & 16 cartridges \\
\hline Nasal spray & $0.5 \mathrm{mg}$ per spray & 40 sprays \\
\hline Sublingual tablets & $2 \mathrm{mg}$ & 40 tablets \\
\hline
\end{tabular}

respiratory tract infections, coughing, and sore throat. ${ }^{3}$ It has also been reported that frequent nicotine exposure caused hyperkeratosis in the hamster cheek pouch. ${ }^{4}$

NiQuitin $\mathrm{CQ}^{\circledR}$ Lozenges are NRT lozenges that come in $2 \mathrm{mg}$ and $4 \mathrm{mg}$ formulations. They are kept in the mouth for up to 30 minutes and patients are instructed to take one lozenge every one to two hours, up to a maximum of 15 lozenges per day for six weeks. Patients are then told to reduce the dose to one lozenge every two to four hours and after ten weeks to one lozenge every four to eight hours. ${ }^{5}$ The treatment period is usually three months.

Apart from nicotine, other ingredients in the lozenges include: mannitol (E421), xanthan chewing gum (E415), potassium bicarbonate(E501), calcium polycarbophil, sodium alginate (E401), sodium carbonate (E500), aspartame (E951), magnesium stearate (E572) and the mint flavoured type contain mint flavour and menthol. None of these additives have been know to cause intraoral hyperkeratosis.

This patient remained on $122 \mathrm{mg} \mathrm{loz-}$ enges per day for approximately three years prior to developing the lesion (Fig. 1). Once the dosage was reduced (currently on ten lozenges per day) and the patient started keeping the lozenges in different intraoral sites, the lesion started to resolve (Fig. 3).

\section{COMMENT}

Chronic low grade chemical irritation from NRT lozenges, particularly if kept in the same site intraorally, should be included in the differential diagnosis of white patch lesions of the oral mucous membrane.

The authors would like to thank Dr Keith Hunter and Dr Allan McPhaden from the oral pathology unit Glasgow for providing the histology report and photomicrograph used in this case report.

1. Burton S L, Gitchell J G, Shiffman S. Centers for Disease Control and Prevention (CDC). Use of FDA-approved pharmacologic treatments for tobacco dependence - United States, 1984-1998. MMWR Morbid Mortal Wkly Rep 2000; 49: 665-668.

2. Silagy $\mathrm{C}$, Lancaster $\mathrm{T}$, Stead L et al. Nicotine

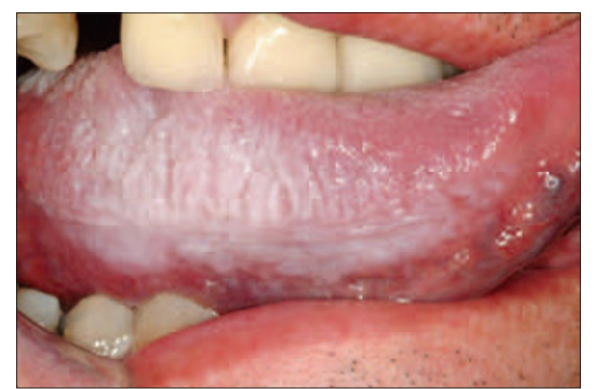

Fig. 1 Clinical picture of keratinisation on right lateral border of the tongue at presentation

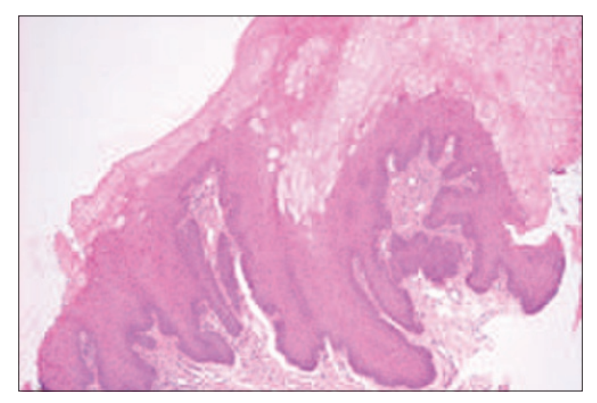

Fig. 2 Haematoxylin and eosin-stained histological section of the lesion (x 200)

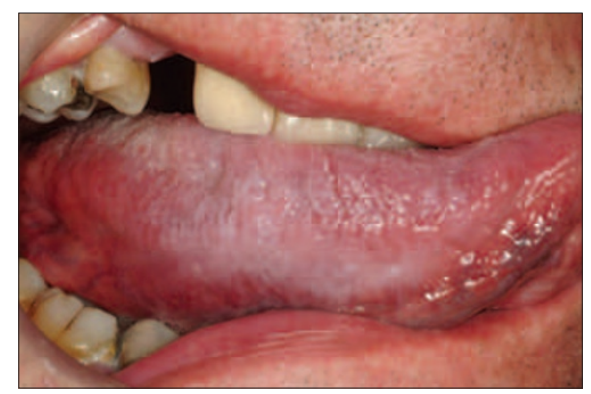

Fig. 3 Clinical picture of right lateral border of tongue after patient attempted to avoid keeping nicotine replacement tablets in right lingual sulcus

replacement therapy for smoking cessation. Cochrane Database Syst Rev 2004; 3: CD000146. 3. Shiffman S, Dresler C M, Hajek P et al. Efficacy of a nicotine lozenge for smoking cessation. Arch Intern Med 2002; 162: 1267-1276.

4. Chen Y P, Johnson G K, Squier C A. Effects of nicotine and tobacco-specific nitrosamines on hamster cheek pouch and gastric mucosa. J Oral Pathol Med 1994; 23: 251-255.

5. NiQuitin $\mathrm{CQ}^{\circledR}$, Click2Quit, GlaxoSmithKline group of companies (Online). Available from http://www click2quit.co.uk/c2q_package/COP02Aii.asp (Accessed 29 January 2007). 